\title{
Quantum computer: an appliance for playing market games
}

\author{
Edward W. Piotrowski \\ Institute of Theoretical Physics, University of Białystok, \\ Lipowa 41, Pl 15424 Białystok, Poland \\ e-mail: ep@alpha.uwb.edu.pl \\ Jan Sładkowski \\ Institute of Physics, University of Silesia, \\ Uniwersytecka 4, Pl 40007 Katowice, Poland \\ e-mail: sladk@us.edu.pl
}

\begin{abstract}
Recent development in quantum computation and quantum information theory allows to extend the scope of game theory for the quantum world. The authors have recently proposed a quantum description of financial market in terms of quantum game theory. The paper contain an analysis of such markets that shows that there would be advantage in using quantum computers and quantum strategies.
\end{abstract}

PACS numbers: 02.50.Le, 03.67.-a, 03.65.Bz, 06.90.+v, 03.67.-a

Keywords: quantum games, quantum strategies, quantum computations, quantum circuits, econophysics, financial markets

\section{Motivation}

Most of reasonable people find their best ways of doing something by analysis of previously met situations. This rule usually results in moves that are profitable or beneficial in a more or less general sense. Sciences and martial arts have worked out an additional nonconservative scheme that consist 
in analysis of the events in their reversed historical perspective (one revises the axioms or decisions bearing their consequences in mind). Shall we believe that traders active on the future markets who would have sophisticated achievement of quantum technology at their disposal would refrain from using them [1]? Below we will discuss some selected aspects of quantum markets [2]-[4] that may form the basis for extraordinary profits. By changing some of our habits we might be able to gain unique profits resulting from discoveries made by researchers in fundamental natural phenomena that suggest more effective ways of playing games [5]. These "new games" cannot by itself create extraordinary profits or multiplication of goods but the dynamism of transaction they may cause would result in more effective markets and capital flow into hands of the most efficient traders. Sophisticated technologies that are not yet available are not necessary to put such a market in motion. Simulation of such markets can be performed in an analogous way to precision physical measurements during which classical apparatuses are used to explore quantum phenomena. People seeking after excitement would certainly not miss the opportunity to perfect their skills at using "quantum strategies". To this end an automatic game "Quantum Market" will be sufficient. And such a device can be built up due to the recent advances in technology.

\section{Interference of traders decisions}

Let us consider trading in some commodity $\mathfrak{G}$ according to as simple as possible but quantum decision rules $[1,2,5]$. The analyzed below game is certainly feasible with contemporary physical instruments but we refrain from stating what the necessary technical requirements for implementation of such games are. Let the states $|0\rangle$ and $|\mathrm{I}\rangle$ denote strategies that the trader selling $\mathfrak{G}$ accepts a low and a high price, respectively [2]. The family $\{|z\rangle\}, z \in \overline{\mathbb{C}}$ of complex vectors (states) $|z\rangle:=|0\rangle+z|\mathrm{I}\rangle(| \pm \infty\rangle:=|\mathrm{I}\rangle)$ represents all trader strategies in the linear hull spread by the vectors $|0\rangle$ and $|\mathrm{I}\rangle$. The coordinates of the vector $|z\rangle$ (we will often call it a qubit to follow the quantum information theory convention) in the basis $(|0\rangle,|\mathrm{I}\rangle)$ give after normalization probability amplitudes of the corresponding traders decisions. It is convenient to identify the strategies $|z\rangle$ with points of the two dimensional sphere $S_{2} \simeq \overline{\mathbb{C}}$. In that case we can use the geographic coordinates $(\varphi, \theta)$ where $\varphi:=\arg z$ and $\theta:=\arctan |z|$. According to the quantum model of market $[2,6,7]$ the strategy $|z\rangle$ expressed in the basis 
$\left(\left|0^{\prime}\right\rangle,\left|\mathrm{I}^{\prime}\right\rangle\right)$ that consists of Fourier transforms of the vectors $(|0\rangle,|\mathrm{I}\rangle)^{1}$

$$
|z\rangle=|0\rangle+z|\mathrm{I}\rangle=\left|0^{\prime}\right\rangle+\frac{1-z}{1+z}\left|\mathrm{I}^{\prime}\right\rangle
$$

describes the trader's decisions concerning buying the commodity $\mathfrak{G}$. In that sense the demand aspects of trader's behavior are Fourier representation of her "supplying preferences". The involutive homography $\mathcal{F}:|n\rangle \rightarrow\left|n^{\prime}\right\rangle=$ $\frac{1}{\sqrt{2}} \sum_{m=0}^{\mathrm{I}}(-1)^{\langle n \mid m\rangle}|m\rangle, n=0$, I that describes the Fourier transform in two dimensions has the form of Hadamard matrix in both bases $(|0\rangle,|\mathrm{I}\rangle)$ and $\left(\left|0^{\prime}\right\rangle,\left|I^{\prime}\right\rangle\right): \frac{1}{\sqrt{2}}\left(\begin{array}{rr}1 & 1 \\ 1 & -1\end{array}\right)$. The vectors $\left|0^{\prime}\right\rangle$ and $\left|I^{\prime}\right\rangle$ correspond to the acceptance of a high and a low price of the commodity $\mathfrak{G}$ when buying, respectively. Note that buying $\mathfrak{G}$ at a high price corresponds to selling money at low price expressed in units of $\mathfrak{G}$. The squared absolute value of the number $z$ that parameterizes the trader's strategy

$$
|z\rangle=|0\rangle+|z| \mathrm{e}^{\mathrm{i} \arg (z)}|\mathrm{I}\rangle
$$

has an orthodox stochastic (non-quantum) interpretation as a relative measure of the probability of the event (selling at high price) with respect to the alternative event (selling at low price) that happens with weight 1 . The phase $\varphi:=\arg (z)$ of the parameter $z$ is characteristic of the quantum description. For $\varphi=0$ the absolute value of $\frac{1-z}{1+z}$ reach its minimal value $\frac{|1-| z||}{1+|z|}$ and for $\varphi=\pi$ the maximal one, equal to the inverse of the minimal value. For $\varphi=\frac{\pi}{2}$ and $\varphi=\frac{3 \pi}{2}$ the absolute value of $\frac{1-z}{1+z}$ is equal to one, what corresponds to equal probabilities $\frac{\left|\left\langle z \mid 0^{\prime}\right\rangle\right|^{2}}{\langle z \mid z\rangle}$ and $\frac{\left|\left\langle z \mid I^{\prime}\right\rangle\right|^{2}}{\langle z \mid z\rangle}$. Changes in the phase $\varphi$ produce no effect on the probabilities of selling but may change the probabilities of buying corresponding to the Fourier transformed strategies. Various price preferences of the selling the commodity $\mathfrak{G}$ trader may interfere and influence on his behavior as a buyer. Analogous observation can be made about the phase of the strategy represented in the basis $\left(\left|0^{\prime}\right\rangle,\left|I^{\prime}\right\rangle\right.$ ). (This should be compared with behavior according to signals given by various tools used in technical analysis.) In that way one quantum strategy may "compete" with two independent "classical" strategies. This mechanism enable extraordinary profits that can hardly be achieved in "the classical way". Quantum strategies have also other interesting properties. For example for games that can

\footnotetext{
${ }^{1}$ The bases $\left(\left|0^{\prime}\right\rangle,\left|I^{\prime}\right\rangle\right)$ and $(|0\rangle,|I\rangle)$ exemplify the notion of conjugate bases that correspond to "extreme" nonmeasurable simultaneously observables in a finite dimensional Hilbert space, cf [8].
} 
be represented in countably dimensional Hilbert spaces the maximal profit is achieved at a fixed point of the tactics what gives an effective method of adopting strategies to the continuously changing market situation [4].

\section{Non-collective quantum tactics}

The elementary tactics of a trader are those that result in inverted behavior: selling (buying) at low price is switched to selling (buying) at high price and vice versa. Such change in the supply strategy is described in the basis $(|0\rangle,|\mathrm{I}\rangle)$ by the Pauli matrix $\sigma_{1}$

$$
\mathcal{X}=\left(\begin{array}{ll}
0 & 1 \\
1 & 0
\end{array}\right),
$$

$\mathcal{X}^{2}=I$. Analogously the change in the demand strategy is described by the Pauli matrix $\sigma_{3}$

$$
\mathcal{X}^{\prime}=\left(\begin{array}{rr}
1 & 0 \\
0 & -1
\end{array}\right)
$$

because $\mathcal{X}^{\prime}=\mathcal{F} \mathcal{X} \mathcal{F}$. The matrices $\mathcal{X}$ and $\mathcal{X}^{\prime}$ are interchanged by Fourier transform therefore their sum is invariant. We have $\mathcal{F}=\frac{1}{\sqrt{2}}\left(\mathcal{X}+\mathcal{X}^{\prime}\right)$ in both bases. This means that for given probabilities of switching supply to demand $\mathcal{F}$ represents a tactic. We will call any linear operation transforming a single strategy (i.e. a qubit) a non-collective tactic (a quantum gate). To illustrate the importance of the demand-supply symmetry of the tactics $\mathcal{X}$ i $\mathcal{X}^{\prime}$ let us consider the change of the tactics $|z\rangle$ connected with the problem of finding out if some unknown function $g_{\text {? }}$ belonging to the class $g_{k}:\{0,1\} \rightarrow\{0,1\}$, $k=0, \ldots, 3$ is constant. To this end it is sufficient to restrict oneself to the tactics $\mathcal{G}$ ? changing sign of the homogenous coordinates of the strategy $|z\rangle$. In the supply basis we have:

$$
\mathcal{G}_{k}|z\rangle:=(-1)^{g_{k}(0)}|0\rangle+(-1)^{g_{k}(1)} z|\mathrm{I}\rangle .
$$

For the constant functions $g_{0}(0)=g_{0}(1)=0$ and $g_{1}(0)=g_{1}(1)=1$ the respective involutions comprise of the identities $\mathcal{G}_{0}=\mathcal{G}_{1}=I$ and for the remaining functions $g_{2}(0)=g_{3}(1)=0$ and $g_{3}(0)=g_{2}(1)=1$ we have the inversion of buying strategies, $\mathcal{G}_{2}=\mathcal{G}_{3}=\mathcal{X}^{\prime}$. Therefore we have to determine if the use of the tactic $\mathcal{F} \mathcal{G}_{k} \mathcal{F}$, equal to $I$ for $k=0,1$ and $\mathcal{X}$ for $k=2,3$, changes the supply strategies $|0\rangle$ or $|\mathrm{I}\rangle$. This simple method of deciding the question 
by simultaneous action of the tactics $\mathcal{G}_{\text {? }}$ on the superposition of two basic supply strategies $\left(\mathcal{F}|0\rangle=\left|0^{\prime}\right\rangle=|0\rangle+|\mathrm{I}\rangle\right)$ is known as the Deutsch Oracle [9]. This simple example, although of academic concern, shows the advantage of quantum strategies over classical ones. One have to determine only one of the possible values of $g_{\text {? }}$ to execute the strategy $\mathcal{G}_{\text {? }}$ because it simultaneously acts in the parallel universes. The non-quantal method of identification of the label $k$ of the function $g_{k}$ is not so quick because it requires previous cataloguing (ie measuring) of all functions $g_{0}, g_{1}, g_{2}, g_{3}$.

Any tactic can be expressed in terms of Pauli matrices because the second generator of the algebra $\mathrm{su}(2), \sigma_{2}$, represents the tactic $\mathcal{X} \mathcal{X}^{\prime}$ (in the basis $(|0\rangle,|\mathrm{I}\rangle))$. The stereographic projection $\overline{\mathbb{C}} \rightarrow S_{2}$ can be readily inversed: $S_{2} \ni\left(x_{1}, x_{2}, x_{3}\right)=E_{z}(\vec{\sigma})$, where the vector $E_{z}(\vec{\sigma})=\frac{\langle z|\vec{\sigma}| z\rangle}{\langle z \mid z\rangle}$ represents the expectation value of the vector of Pauli matrices $\vec{\sigma}:=\left(\sigma_{1}, \sigma_{2}, \sigma_{3}\right)$ for a given strategy $|z\rangle$. Due to this relation to the $S U(2)$ group these tactics are represented in terms of special unitary matrices and can be parameterized by an element of $\overline{\mathbb{C}}$ and an angle $\alpha \in[0, \pi]$ :

$$
S U(2) \ni \mathcal{U}_{z, \alpha}=\mathrm{e}^{\mathrm{i} \alpha \vec{\sigma} \cdot E_{z}(\vec{\sigma})}=I \cos \alpha+\mathrm{i} \vec{\sigma} \cdot E_{z}(\vec{\sigma}) \sin \alpha .
$$

The coordinates $\left(\cos \alpha, E_{z}(\vec{\sigma}) \sin \alpha\right) \in \mathbb{R}^{4}$ are in $1-1$ correspondence with the sphere $S_{3}\left(\cos ^{2} \alpha+E_{z}^{2}(\vec{\sigma}) \sin ^{2} \alpha=1\right)$. The transition from the supply picture to the demand picture is given by the homography (1):

$$
\mathcal{U}_{z, \alpha} \longrightarrow \mathcal{U}_{z, \alpha}^{\prime}:=\mathcal{F} \mathcal{U}_{z, \alpha} \mathcal{F}=\mathcal{U}_{\frac{1-z}{1+z}, \alpha} .
$$

The property (3) distinguish the coordinates $(z, \alpha)$ of a q-tactics from any other parameterization of $S_{3}$. The above "market interpretation" of the group $S U(2)$ stems from Orlov approach to modelling of consciousness [10, 11]. But there are important differences. The operator $\mathcal{X}^{\prime}$ describes the state of consciousness related to subjective doubts (doubt state) concerning the truth of a given statement and the respective classical logic is represented by some orthonormal basis in $\mathbb{C}^{2}$ (homogeneous coordinates of a qubit) [10]. Our interpretation exposes the operational properties of $\mathcal{X}^{\prime}$ (and its dual $\mathcal{X}$ ) that allow the trader to completely change her demand behavior without influencing her supplying strategy. This result in quantum game theory is related to the construction of transactional logic in which the values truth or false rate utility of a given strategy measured by the respective payoffs. This utilitarian relativism is closely related to Deutsch philosophy of science [12]. 


\section{Wigner function of a quantum strategy}

To present the effectiveness of quantum market strategies we restrict our analysis to the most profitable situation of a game against a "non-quantum" market with equal buying and selling prices. Besides we will suppose that buying and selling transaction intensities are equal. This means that the transaction are specified by equally frequent measurements of both polarizations $|0\rangle$ and $\left|0^{\prime}\right\rangle[2,6]$. The case of non-equal transaction intensities can be analyzed in an analogous way by means of quantum tomography [13]. If we suppose that there are only two price levels (low and high) and the payoffs are 1,0 or -1 (gain - no transaction - loss) then the payoff matrix takes the form given in Table 1. For example, if the price of the commodity $\mathfrak{G}$ is

\begin{tabular}{r|r|r|r|c|}
\multicolumn{1}{c}{$00^{\prime}$} & \multicolumn{1}{c}{$01^{\prime}$} & \multicolumn{1}{c}{$10^{\prime}$} & $11^{\prime}$ \\
\cline { 2 - 5 } low price & $-1+1=0$ & $-1+1=0$ & $0+1=1$ & $0+1=1$ \\
\cline { 2 - 5 } high price & $1-1=0$ & $1+0=1$ & $1-1=0$ & $1+0=1$ \\
\cline { 2 - 5 } & &
\end{tabular}

Table 1: The payoff matrix (trader's gains).

low then the trader whose strategy is sell at low price $(|0\rangle)$ and buy at high price $\left(|0\rangle^{\prime}\right)$ loses when selling (payoff is -1 ) and gains when buying (payoff is 1) because being ready to buy at high price she certainly will buy at low price. The average payoff is $-1+1=0$. From the classical point of view the optimal solution is to use two independent strategies: sell at high price and buy at low price. This results in average payoff $\frac{1}{2}$ per transaction. What is the optimal strategy in the quantum domain? To find out we should determine a measure of intertwining of all possible trader's moves. This can be done with help of the Wigner function formalism. The Wigner function for the strategy $|z\rangle$ has the form of a $2 \times 2$ matrix [14]

$$
W_{k m^{\prime}}\left(\mathrm{e}^{\mathrm{i} \varphi} \tan \theta\right)=\frac{1}{4}\left(1+(-1)^{k} \cos 2 \theta+(-1)^{m^{\prime}} \sqrt{2} \sin 2 \theta \cos \left(\varphi-(-1)^{k} \frac{\pi}{4}\right)\right),
$$

$k, m^{\prime}=0,1$. The function $W_{k m^{\prime}}(z)$ gives the measure (not always positive definite) of the state $|z\rangle$ being simultaneously in both states $(1-k)|0\rangle+k|\mathrm{I}\rangle$ and $\left(1-m^{\prime}\right)\left|0^{\prime}\right\rangle+m^{\prime}\left|\mathrm{I}^{\prime}\right\rangle$. The respective probabilities of measurements of the 
strategy being in the states $|0\rangle,|\mathrm{I}\rangle,\left|0^{\prime}\right\rangle,\left|\mathrm{I}^{\prime}\right\rangle$ are :

$$
\begin{gathered}
W_{00}(z)+W_{01}(z)=\frac{|\langle z \mid 0\rangle|^{2}}{\langle z \mid z\rangle}, W_{10}(z)+W_{11}(z)=\frac{|\langle z \mid \mathrm{I}\rangle|^{2}}{\langle z \mid z\rangle}, \\
W_{00}(z)+W_{10}(z)=\frac{\left|\left\langle z \mid 0^{\prime}\right\rangle\right|^{2}}{\langle z \mid z\rangle}, W_{01}(z)+W_{11}(z)=\frac{\left|\left\langle z \mid \mathrm{I}^{\prime}\right\rangle\right|^{2}}{\langle z \mid z\rangle} .
\end{gathered}
$$

These sums being measurable in "experiment" should be nonnegative. The same is true for the sums of diagonal elements of the matrix $W(z)$ because they give probabilities of measurement of strategies $|i\rangle$ and $|-i\rangle$ :

$$
W_{00}(z)+W_{11}(z)=\frac{|\langle z \mid \mathrm{i}\rangle|^{2}}{\langle z \mid z\rangle\langle\mathrm{i} \mid \mathrm{i}\rangle}, W_{10}(z)+W_{01}(z)=\frac{|\langle z \mid-\mathrm{i}\rangle|^{2}}{\langle z \mid z\rangle\langle-\mathrm{i} \mid-\mathrm{i}\rangle} .
$$

This means sum of any two entries of the matrix $W(z)$ is nonnegative. It follows that only one of the entries of the matrix $W(z)$ can be negative. There is a simple "quantum" strategy that outperforms any "classical" one: one should look for such strategies $|z\rangle$ that the negative element of the matrix $W(z)$ corresponds to the minimal element of the payoff matrix. Fig. 1 presents regions of the parameter space $\left(S_{2}\right)$ that are not accessible to a "classical player". The white regions correspond to non positive definite matrices $W\left(\mathrm{e}^{\mathrm{i} \varphi} \tan \theta\right)$. Their boundaries form four circles that correspond to the families of strategies $z(\phi)= \pm\left(1+(1-\mathrm{i}) \tan \frac{\phi}{2}\right)$ i $z(\phi)= \pm \frac{1}{2}\left(\sqrt{2} \mathrm{e}^{\mathrm{i} \phi}+1-\mathrm{i}\right)$, $\phi \in[-\pi, \pi)$. If we use a measure on $S_{2}$ that is invariant with respect to q-tactics then the area of the classically inaccessible region is maximal. In that sense the quantum approach is the maximal one. It is tempting to define a measure $\kappa$ of non-positivity of the Wigner function that causes the attractiveness of quantum strategies. If we use the minimal entry of the matrix $W(z)$ to this end we get $\kappa(\varphi, \theta):=-\min _{k, l} W_{k l}(\varphi, \theta)$ for regions where $W(z)$ is not positive definite and $\kappa(\varphi, \theta):=0$ otherwise. Fig. 2 presents the sphere of trader's strategies of radius $r$ modified so that this factor is stressed $(r \rightarrow(1+2 \kappa(\varphi, \theta)) r)$.

\section{Non-collective game against market dictat- ing prices}

Let $p$ be the probability that the market bids a high price for the asset $\mathfrak{G}$ and $1-p$ be the probability of a low bid. If payoff matrix is given by Table 


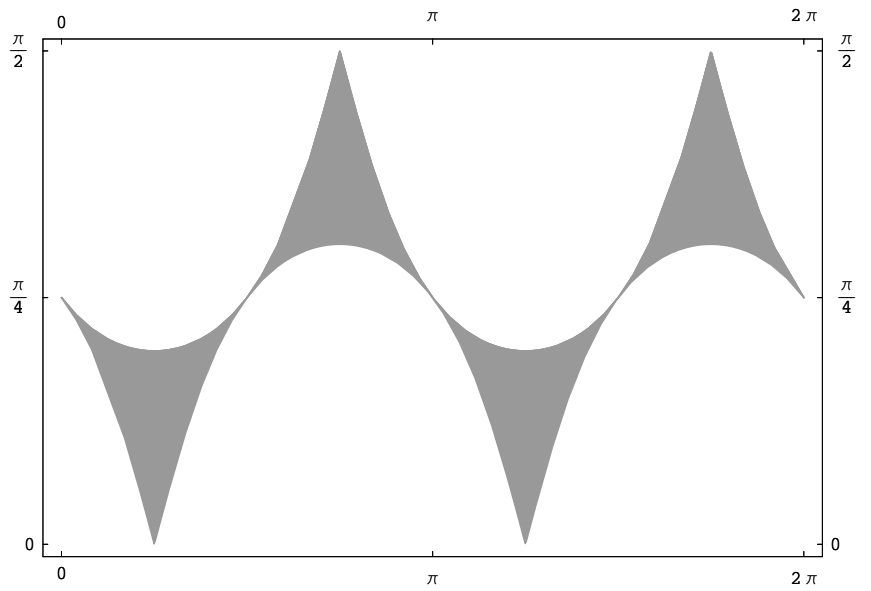

Figure 1: Geographic map of quantum strategies. The upper curve corresponds to two circles tangent at the north pole and are given by the formula $\theta(\varphi)=\frac{1}{2} \arccos \frac{\sin 2 \varphi}{2+\sin 2 \varphi}$.

1 then the trader's average payoff is

$$
\begin{aligned}
w(p, z):= & E(M)=(1-p)\left(W_{10^{\prime}}+W_{11^{\prime}}\right)+p\left(W_{01^{\prime}}+W_{11^{\prime}}\right)= \\
& p\left(W_{01^{\prime}}-W_{10^{\prime}}\right)+W_{10^{\prime}}+W_{11^{\prime}}=\frac{p(1-z)(1-\bar{z})+2(1-p)|z|^{2}}{1+|z|^{2}} .
\end{aligned}
$$

The strategy that maximizes the expected profit $w(p, z)$ is, except for $p=0$ or 1 , a giffen $[2,15]$ that is it does not comply with the law of demand and supply ${ }^{2}$ :

$$
z_{\max }(p)=\frac{1-p}{p}-\sqrt{1+\left(\frac{1-p}{p}\right)^{2}} .
$$

This family of strategies comprises one fourth of a circle $\varphi=\pi, \theta \in\left[\frac{\pi}{4}, \frac{\pi}{2}\right]$ in the parameter space $S_{2}$. For $p=\frac{1}{2}$ we have an optimal strategy with maximal value of $\kappa$ with Wigner matrix of the form

$$
W\left(\pi, \frac{3}{8} \pi\right)=\frac{1}{4}\left(\begin{array}{cc}
1-\sqrt{2} & 1 \\
1 & 1+\sqrt{2}
\end{array}\right) .
$$

\footnotetext{
${ }^{2}$ The conditional function of demand (supply) that corresponds to the Wigner function formally comply with the demand and supply law but is not measurable because it can be greater than one.
} 


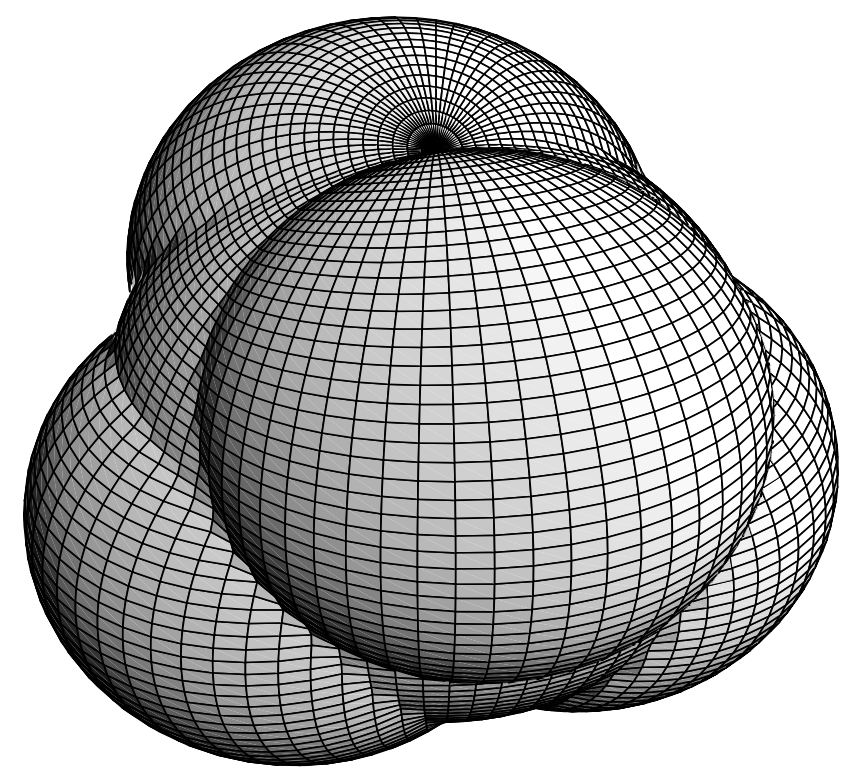

Figure 2: Parameter space $\left(S_{2}\right)$ with radius modified by the $\kappa$ measure of non positivity of Wigner function describing strategies. The maximal "departure" is achieved for the strategy $z= \pm\left(\frac{\sqrt{3}-1}{2}(1-i)\right)^{ \pm 1}$.

This strategy corresponds to a fixed point of the tactics $\mathcal{X} \mathcal{X}^{\prime} \mathcal{F} \mathcal{X}^{\prime} \mathcal{X}$. Let us notice that in this case the worst quantum strategy is the one that has the same form in both representations, $z=\sqrt{2}-1$ (profit is equal to $\frac{2-\sqrt{2}}{4}$ ). Nevertheless it is better than any classical strategy.

The game discussed above can be easily generalized to a realistic case without restrictions on the price of $\mathfrak{G}$. The resulting quantum market can be perceived as a sum of games (with qubit strategies) for all binary digits of the logarithm of the price of $\mathfrak{G}$. The use of logarithms makes the considerations independent of monetary units and units used to measure the commodity $\mathfrak{G}$.

\section{Alliances}

Let us now consider a classical market organized in such a way that the strategies can influence each other. Suppose that there are $N$ active players. The 


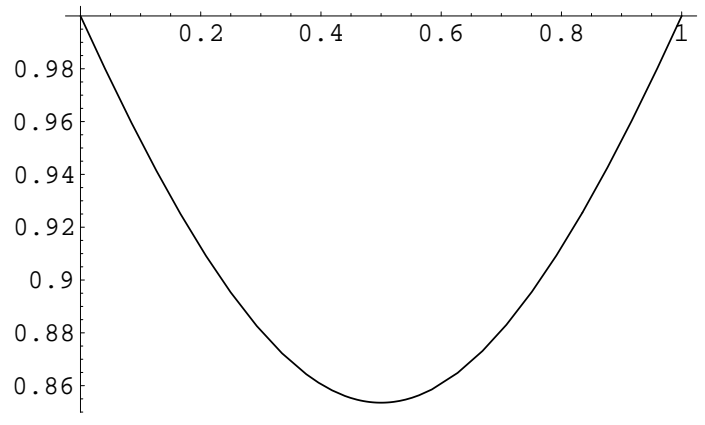

Figure 3: Maximal profit as a function of intensity $p$ of high price bids, $w_{\max }=\frac{1}{2}+\sqrt{\frac{1}{8}+\frac{1}{2}\left(p-\frac{1}{2}\right)^{2}}$ (in a classical game maximal profit is $\frac{1}{2}$ ).

state of the game is represented by an element of $\mathbb{C} P^{2^{N}-1}$. The homogeneous coordinates are formed by tensor product od the qubits' coordinates of separate players. Such a market can be formed for example by sufficiently large statistical ensemble of collective strategies supplemented with an appropriate clearinghouse that measures supply and demand and sets the "optimal" prices. The analysis of such markets is a challenge but the properties of unitary transformations suggest that the task can be performed with help of quantum computation [16]. In a quantum market game [2] any $N$-qubit unitary transformation seems to be acceptable as a collective tactic because it can be easily realized in terms of simple operations performed by individual players or pairs of players. To this end it suffices to define a two qubit involutive gate $\mathcal{C}$

$$
\mathcal{C}:=\frac{1}{2}\left(I+\mathcal{X}^{\prime}\right) \otimes I+\frac{1}{2}\left(I-\mathcal{X}^{\prime}\right) \otimes \mathcal{X}
$$

that is nontrivial only on a subspace corresponding to two arbitrary selected players. If the first player is ready to sell $\mathfrak{G}$ only at high price (strategy $|\mathrm{I}\rangle)$ then the operation $\mathcal{C}$ inverses the second player's strategy. It leaves it untouched if the first player accepts low price when selling. We will call the gate $\mathcal{C}$ an alliance regardless of the standard name controlled-NOT (the analysis given below would explain this apostasy). If we consider the effects of $\mathcal{C}$ in demand representation (in the basis $\left.\left(\left|0^{\prime}\right\rangle\right),\left|\mathrm{I}^{\prime}\right\rangle\right)$ ) then the players roles 
are reversed what follows from:

$\mathcal{C}^{\prime}=(\mathcal{F} \otimes \mathcal{F})\left(\frac{1}{2}\left(I+\mathcal{X}^{\prime}\right) \otimes I+\frac{1}{2}\left(I-\mathcal{X}^{\prime}\right) \otimes \mathcal{X}\right)(\mathcal{F} \otimes \mathcal{F})=I \otimes \frac{1}{2}\left(I+\mathcal{X}^{\prime}\right)+\mathcal{X} \otimes \frac{1}{2}\left(I-\mathcal{X}^{\prime}\right)$

Therefore to tell who is manipulated and who manipulates depends on the demand/supply context. Most of quantum gates are universal in the sense

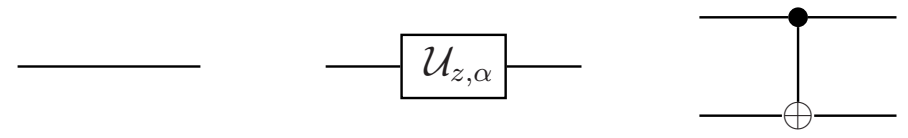

Figure 4: Elements of a quantum scheme (from left to right): strategy, qubit tactic and alliance $\mathcal{C}$.

that any other gate can be composed of a universal one [17]. But for our aims it is more transparent to describe a collective tactic of $N$ players as a sequence of various operations $\mathcal{U}_{z, \alpha}$ performed on one-dimensional subspaces of players' strategies and, possibly, alliances $\mathcal{C}$ among them (any element of $S U\left(2^{N}\right)$ can be given such a form [18]). Therefore an alliance is the only way to form collective games ( $\mathcal{C}$ is universal on one qubit subsystem). All elements necessary for implementation of such games are presented in Figure 4. Description of cases with arbitrary number of commodities or allowed prices is obvious but results in more complex circuits. Note that players can exchange strategies with each other and therefore any permutation of strategies is possible. Exchange of strategies can be accomplished by performing three successive alliances $\mathcal{T}=\mathcal{C} \mathcal{C}^{\prime} \mathcal{C}$, cf Fig. 5. Therefore Eq. (5) can be written in the simple form: $\mathcal{C}^{\prime}=\mathcal{T C} \mathcal{T}$. Consider a player who accepts low price for the

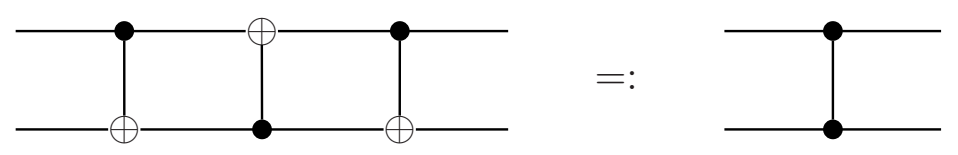

Figure 5: Tactic resulting in exchange of strategies $\mathcal{T}$ (three successive alliances).

commodity she wants to sell. Some other player can use the alliance $\mathcal{C}$ to change her strategy so that the resulting correlated strategy is a two qubit 
entangled strategy

$$
\mathcal{C}|z\rangle|0\rangle=\mathcal{C}|0\rangle|0\rangle+z \mathcal{C}|\mathrm{I}\rangle|0\rangle=|0\rangle|0\rangle+z|\mathrm{I}\rangle|\mathrm{I}\rangle
$$

Strategies of selling at high prices can be $\mathcal{C}$-transformed into anti-correlated entangled strategy

$$
\mathcal{C}|z\rangle|\mathrm{I}\rangle=|0\rangle|\mathrm{I}\rangle+z|\mathrm{I}\rangle|0\rangle
$$

Analogous manipulations (transposition) are possible in the demand part of the market.

\section{Alliances: measurement and interference}

An alliance allows the player to determine the supply or demand state of another player by making an alliance and measuring her resulting strategy. This process is shortly described as

$$
\mathcal{C}\left|0^{\prime}\right\rangle\left|m^{\prime}\right\rangle=\left|m^{\prime}\right\rangle\left|m^{\prime}\right\rangle, \quad \mathcal{C}|m\rangle|0\rangle=|m\rangle|m\rangle,
$$

where $m=0$, I. The corresponding diagrams are shown in Fig. 6. The left diagram presents measurement of the observable $\mathcal{X}$ and the right one measurement of $\mathcal{X}^{\prime}$. Any measurement would demolish possible entanglement
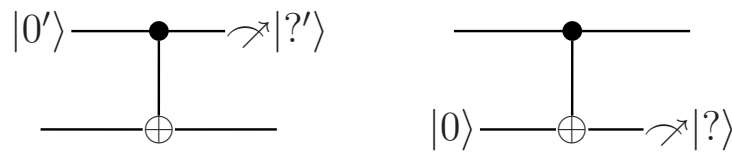

Figure 6: Alliance as a means of determining others' strategies. The sign " $>$ " at the right ends of lines representing qubits symbolizes measurement.

of strategies. Therefore entangled quantum strategies can exist only if the players in question are ignorant of the details of their strategies. Let us now consider an interesting version of the game when the players have "quantum minds" (or are sort of self-conscious quantum automata [19]). According to the many worlds interpretation of quantum theory [12] such a player is aware of her strategy belonging to any of the interfering worlds. If the worlds do not decouple for some time and strategies are transformed according to the applied tactics and if eventually the measurement is performed then such a 
player would not be aware of the decoupled histories. Any quantum mind that wants to evolve in a unitary (deterministic) quantum way is involuntary as being condemned to a quantum evolution (cf [20] for a discussion of the free choice problem). Such a quantum mind may try to influence his evolution by a tactics profitable from the point of view of that component of the interfering strategies that she is aware of. But such operation announce information about her (quantum) state and is equivalent to a measurement that would destroy any extraordinary profits resulting from interference with other worlds. However, the possibility of choosing between destroying and preserving the interference alone gives her certain advantages over the classical minds. Only passive persistence in the interference due to the necessary amnesia would postpone the eventual valuation of her behavior. Details of conclusions one can draw may depend on the actual interpretation of quantum theory one is ready to accept. Nevertheless, parties playing a quantum game must be very careful in controlling their strategies and the very measurement should form an inseparable ingredient of tactic/strategy. Therefore the limited knowledge of players' strategy result in a characteristic for a quantum game spontaneousness. Eq. (2) and the identities:

$$
\begin{aligned}
(I \otimes \mathcal{X}) \mathcal{C} & =\mathcal{C}(I \otimes \mathcal{X}) \\
\left(\mathcal{X}^{\prime} \otimes I\right) \mathcal{C} & =\mathcal{C}\left(\mathcal{X}^{\prime} \otimes I\right), \\
(\mathcal{X} \otimes I) \mathcal{C} & =\mathcal{C}(\mathcal{X} \otimes \mathcal{X}) \\
\left(I \otimes \mathcal{X}^{\prime}\right) \mathcal{C} & =\mathcal{C}\left(\mathcal{X}^{\prime} \otimes \mathcal{X}^{\prime}\right)
\end{aligned}
$$

imply that any game (collective strategy) can be perceived as superposition of some simpler collective strategies that result from two stages (one can reverse the time arrow). First, players form alliances and then some of them inverse supply $\mathcal{X}, \mathcal{X}^{\prime}$ or both $\mathcal{X} \mathcal{X}^{\prime}$. Transposition of alliances and qubit inversions does not change anything or results in cloning of qubits.

The following digressions seems to be in place here. In 1970 Sthephen Wiesner invented counterfeit-proof (quantum) money [8]. The idea was based on the first proposal of a one-way function that exploits unique properties of quantum states. This proposal contrary to its mathematical counterparts [21] cannot be questioned because fundamental laws of Nature guarantee its properties. Wiesner's proposal was put forward three years earlier than Cock's idea of using difficult to reverse operations in cryptography (cf the construction of safe quantum cryptography by Bennett and Brassard in 1984 
that cannot be broken even with the help of quantum computers) [22]. According to Wiesner a counterfeit-proof banknote should have two numbers, one of them is kept secret. The secret number is formed from two random binary sequences and is encoded in a two-state quantum systems as one of the four states $|0\rangle,|\mathrm{I}\rangle,\left|0^{\prime}\right\rangle$ and $\left|\mathrm{I}^{\prime}\right\rangle$ in accordance with a scheme presented in [8]. The second number plays the role of an ordinary serial number. The knowledge of the two randomly generated binary sequences allows to perform a non-destroying verification (measurement) of the authenticity of the note (the proper method of reading of the qubits forms the trap-door of Wiesner's one-way function). The demand or supply formal aspect of the binary digit requires a correct method of measurement (left of right part in Fig. 6). Wiesner's pioneering idea of quantum banknote and Deutsch Oracle show that the mathematical notion of a function might be more realistic than one is ready to admit. Present development of quantum information theory is a case in point for creativity of such attitude. Properties of quantum world may seem to be strange but always comply with objective laws of Nature.

\section{Some remarks on collective market games}

A game when only "classical" tactics $\mathcal{T}$ are allowed can be used for lotterydrawing when there is no drawing machine available [23]. Such a drawing can have the following form. The person that carries the drawing out (she might also take part in the drawing) draws parallel horizontal lines on a sheet of paper that correspond to the participants. Then she marks the left end of one the lines bends the sheet so that the other participant cannot see the left ends. At that moment the proper drawing begins: every participant draws arbitrary number of vertical lines that join two horizontal lines. The win-

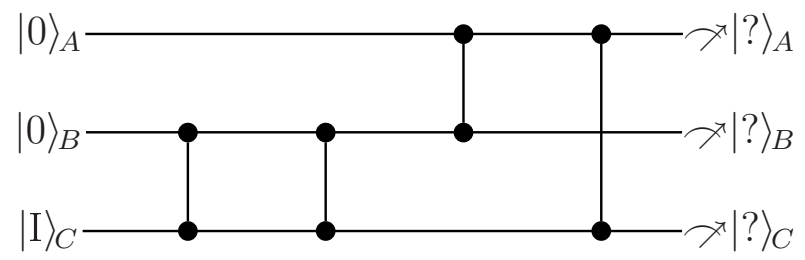

Figure 7: Drawing without drawing machine — the system is built from transpositions $\mathcal{T}$ alone. 
ner is that person whose line's end shows the way out of the maze built up according to the drawing (one starts at the marked point, goes to the right and must go along each vertical line met; going back or turning to the left is forbidden). For example Alice (A) win in the situation presented in Fig. 7. The game is fair because the result is given by a permutation composed from random transpositions.

Let us now consider a collective game that has no classical counterpart. Let as call it Master and pupil. Alice (A) is ready to sell the asset $\mathfrak{G}$ at low price and Bob (B) wants to buy $\mathfrak{G}$ even at high price. But Bob, instead of making the deal (according to the measured strategies), enters into an alliance with Alice. Aftermath Alice changes her strategy according to the tactic (2) and end enters into an alliance with Bob. As a result an entangled

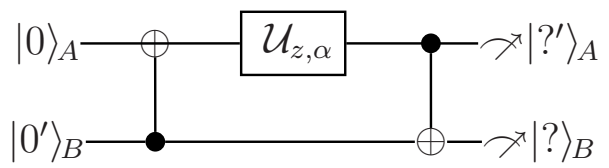

Figure 8: The game Master and pupil (dense coding).

quantum state $|z, \alpha\rangle_{A B} \in \mathbb{R} P^{3} \subset \mathbb{C} P^{3}$ is formed, cf Fig. 8:

$$
|z, \alpha\rangle_{A B}:=\mathcal{C}\left(\mathcal{U}_{z, \alpha} \otimes I\right) \mathcal{C}^{\prime}|0\rangle_{A}\left|0^{\prime}\right\rangle_{B}=
$$

$\cos (\alpha)\left|0^{\prime}\right\rangle_{A}|0\rangle_{B}+\mathrm{i} \sin (\alpha)\left(E_{z}(\mathcal{X})\left|0^{\prime}\right\rangle_{A}|\mathrm{I}\rangle_{B}+E_{z}\left(\mathcal{X}^{\prime}\right)\left|\mathrm{I}^{\prime}\right\rangle_{A}|0\rangle_{B}+E_{z}\left(\mathcal{X} \mathcal{X}^{\prime}\right)\left|\mathrm{I}^{\prime}\right\rangle_{A}|\mathrm{I}\rangle_{B}\right)$

Although Bob cannot imitate Alice tactic $\mathcal{U}_{z, \alpha}$ by simple cloning of the state, he can gather substantial knowledge about her strategy when she is buying (he is able to measure proportions among the components $I, \mathcal{X}, \mathcal{X}^{\prime}$ and $\left.\mathcal{X} \mathcal{X}^{\prime}\right)$. The game is interesting also from Alice point of view because it allows her to form convenient correlations of her strategy with Bob's. Such procedure is called dense coding in quantum information theory [24]. If Alice and Bob are separated from each other and have formed the entangled state $|0\rangle_{A}|0\rangle_{B}+|\mathrm{I}\rangle_{A}|\mathrm{I}\rangle_{B}$ (this is the collective strategy before the execution of $\left.\mathcal{U}_{z, \alpha} \otimes I\right)$ then Alice is able to communicate her choice of tactic $\left(I, \mathcal{X}, \mathcal{X}^{\prime}\right.$, $\mathcal{X} \mathcal{X}^{\prime}$ ) to Bob (bits of information) by sending to him a single qubit. Bob can perform a joint measurement of his and Alice's qubits. Only one of four orthogonal projections on the states $\left|0^{\prime}\right\rangle_{A}|0\rangle_{B},\left|0^{\prime}\right\rangle_{A}|\mathrm{I}\rangle_{B},\left|\mathrm{I}^{\prime}\right\rangle_{A}|0\rangle_{B}$ and $\left|\mathrm{I}^{\prime}\right\rangle_{A}|\mathrm{I}\rangle_{B}$ 
will give a positive result forming the message ${ }^{3}$. Such concise communication is impossible for classical communication channels and any attempt at eavesdropping would irreversibly destroy the quantum coherence (and would be detected).

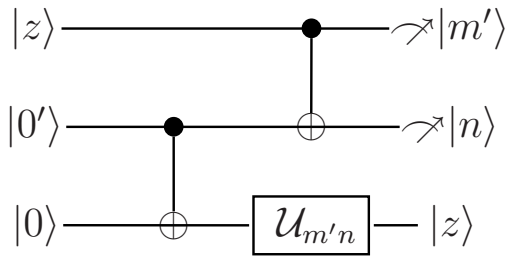

Figure 9: Teleportation of the strategy $|z\rangle$ consisting in measurement of the tactic $\mathcal{U}_{m^{\prime} n}:=\mathcal{X}^{[n=\mathrm{I}]} \mathcal{X}^{\prime\left[m^{\prime}=\mathrm{I}^{\prime}\right]}$ (the notation $[$ true $]:=1$ and $[$ false] $:=0$ is used).

If one player forms an alliance with another that has already formed another alliance with a third player then the later can actually perform measurements that will allow him to transform his strategy to a strategy that is identical to the first player's primary strategy (teleportation [25, 26]). This is possible due to the identity (remember that $\mathcal{X}, \mathcal{X}^{\prime}, \mathcal{X} \mathcal{X}^{\prime}$ are involutive maps)

$2(\mathcal{C} \otimes I)(I \otimes \mathcal{C})|z\rangle\left|0^{\prime}\right\rangle|0\rangle=\left|0^{\prime}\right\rangle|0\rangle|z\rangle+\left|0^{\prime}\right\rangle|\mathrm{I}\rangle \mathcal{X}|z\rangle+\left|\mathrm{I}^{\prime}\right\rangle|0\rangle \mathcal{X}^{\prime}|z\rangle+\left|\mathrm{I}^{\prime}\right\rangle|\mathrm{I}\rangle \mathcal{X} \mathcal{X}^{\prime}|z\rangle$.

Recall that quantum strategies cannot be clonned (no-cloning theorem) but if there are several identical strategies their number cannot be reduced by classical means (no-reducing theorem).

Obviously, the most effective way of playing collective quantum market game is as follows. One have adopt such tactic that transform the initial strategy to one giving maximal profit, $\left|z_{\max }(p)\right\rangle$. It seems that the players alone should decide what forms of tactics are allowed in fact they should construct the whole market. For example, players are allowed to tackle only their own qubits, form a limited number of alliances that can be secret or public. Such an autonomous market should have sort of clearinghouse that besides measuring the respective tactics charges some brokerage.

Collective quantum market games can used for convenient allocation of rules of disposition of the asset $\mathfrak{G}$. In that case the direct aim of a player is to

\footnotetext{
${ }^{3}$ Answers to the questions Would Alice buy at high price? and Would Bob sell at low price? would decode the message.
} 
get an appropriate quantum entanglement instead of maximization of profit. Sort of envy-free rules can also be introduced. For example, an alliance formed at later stages of the game gives substantial advantage. Therefore players that have already gave up forming alliances may be allowed to exchange strategies $\left(\mathcal{C C}^{\prime} \mathcal{C}\right)$ according to the Banach-Knaster protocol [27]. For $N=2$ this protocol is used in fair division: one divides and the other chooses ${ }^{4}$.

The knowledge about the topology of formed alliances influences the actual method of looking for optimal tactics and their form. The investigation of effective methods of playing collective quantum market games is very complicated even in simplified version therefore it will be postponed to a subsequent work.

\section{Conclusions}

Quantum game theory [9],[29]-[31] could have not emerged earlier because a tournament quantum computer versus classical one is not possible without technological development necessary for a construction of quantum computers. Traders active on the markets of future would not hesitate to take the advantage of technological development. The analysis presented here shows that quantum market games or more general quantum-like approach to market description might turn out to be an important theoretical tool for investigation of computability problems in economics or game theory even if never implemented in real markets.

\section{References}

[1] S. Waite, "Quantum investing", Texere Publishing, London (2002).

[2] E. W. Piotrowski, J. Sładkowski, "Quantum Market Games", Physica A 312 (2002) 208.

\footnotetext{
${ }^{4}$ Mediterranean civilizations have been using this method for at least 2800 years [27]. It has been used in popular games in the Far East (http://www.playsite.com). Nowadays it is used in the game of hex invented by Piet Hein in 1942 and reinvented by John Nash. V. Anshelevich has put forward and effective algorithm for playing hex that is equivalent to finding maximal current in Kirchoff's circuit [28] and probably has interesting connections with quantum games.
} 
[3] E. W. Piotrowski and J. Sładkowski, "Quantum-like approach to financial risk: quantum anthropic principle", Acta Phys. Pol. B32 (2001) 3873 .

[4] E. W. Piotrowski, "Fixed Point Theorem for Simple Quantum Strategies in Quantum Market Games", Physica A, in press.

[5] E. W. Piotrowski and J. Sładkowski, "An invitation to quantum game theory", International Journal of Theoretical Physics, in press; quant$\mathrm{ph} / 0211191$.

[6] E. W. Piotrowski, J. Sładkowski, "Quantum Bargaining Games", Physica A 308 (2002) 391.

[7] E. W. Piotrowski, J. Sładkowski, "Quantum English Auctions", Physica A 318 (2003) 505.

[8] S. Wiesner, "Conjugate coding", SIGACT News 15/1, (1983) 78; http://kh.bu.edu/qcl/pdf/wiesners198316024137.pdf.

[9] D. Deutsch, A. Eckert, R. Lupacchini, "Machines, logic and quantum physics", The Bulletin of Symbolic Logic 6 (2000) 265.

[10] Y. F. Orlov, "The wave logic of consciousness: a hypothesis", International Journal of Theoretical Physics, 21 (1982) 37.

[11] T. L. Clarke, D. J. Kaup, "Quantum algorithms for modelling and simulation: A grand challenge for modelling and simulation", proceedings of the conference ICGCMS 2002; http://www.thesimguy.com/GC/WMC02.htm .

[12] D. Deutsch, "The Fabric of Reality", PENGUIN, London (1998).

[13] U. Leonhard, "Discrete Wigner Function and quantum-state tomography", Phys. Rev. A52 (1996) 2998.

[14] A. Miranowicz, W. Leonski, N. Imoto, "Quantum-optical states in finite dimensional Hilbert space", Advances in Chemical Physics 119 (2001) 155.

[15] J. Sładkowski, "Giffen paradoxes in quantum market games", Physica $A$ in press; cond-mat/0211083. 
[16] M. A. Nielsen, I. L. Chuang, "Quantum Computation and Quantum Information", Cambridge University Press, Cambridge (2000).

[17] D. Deutsch, A. Barenco, A. Eckert, "Universality in quantum computation", Proc. R. Soc. London A 449 (1995) 669.

[18] A. Barenco et al., "Elementary gates for quantum computation", Phys. Rev. A 52 (1995) 3457.

[19] D. Z. Albert, "On quantum-mechanical automata", Phys. Lett. 98A (1983) 249.

[20] E. W. Piotrowski, J. Sładkowski, "Quantum solution to the Newcomb's paradox", submitted to Journal of Cognitive Sciences; preprint quant$\mathrm{ph} / 0202074$.

[21] B. Schneier, "Applied Cryptography", Wiley \& Sons, New York (1994).

[22] S. Singh, "The Code Book: The Science of Secrecy from Ancient Egypt to Quantum Cryptography", Fourth Estate, London (1999).

[23] M. Gardner, "New Mathematical Diversions from Scientific American", Gorge Allen \& Unin Ltd, London (1966).

[24] E. Rieffel, W. Polak, "Introduction to quantum computing for nonphysicists", ACM Computing Surveys 32 (2000) 3.

[25] C. Bennet, et al., Teleporting an unknown quantum state via dual classical end Einstein-Podolsky-Rosen channels, Phys. Rev Lett. 70 (1993) 1895.

[26] S. J. Lomonaco Jr., A Rosetta stone for quantum mechanics with an introduction to quantum computation; quant-ph/ 0007045.

[27] S. J. Brams, A. D. Taylor, "Fair division. From cake-cutting to dispute resolution", Cambridge University Press, Cambridge (1996).

[28] V. V. Anshelevich, "A hierachical approach to computer hex", Artificial Intelligence, 134 (2002) 101.

[29] D. Meyer, "Quantum strategies", Physical Review Letters 82 (1999) 1052. 
[30] J. Eisert, M. Wilkens, M. Lewenstein, "Quantum Games and Quantum Strategies", Physical Review Letters 83 (1999) 3077.

[31] E. W. Piotrowski, J. Sładkowski, An invitation to quantum game theory", International Journal of Theoretical Physics, in press; quant$\mathrm{ph} / 0211191$. 